\title{
Medical \& Biological Engineering \& Computing \\ Automatic glycemic regulation for the pediatric population based on switched control and time-varying IOB constraints: an in silico study \\ --Manuscript Draft--
}

\begin{tabular}{|c|c|c|}
\hline Manuscript Number: & \multicolumn{2}{|l|}{ MBEC-D-20-00006 } \\
\hline Full Title: & \multicolumn{2}{|c|}{$\begin{array}{l}\text { Automatic glycemic regulation for the pediatric population based on switched control } \\
\text { and time-varying IOB constraints: an in silico study }\end{array}$} \\
\hline Article Type: & \multicolumn{2}{|l|}{ Original article } \\
\hline Keywords: & \multicolumn{2}{|c|}{ Artificial pancreas; Switched control; Insulin on board; Constrained control } \\
\hline Corresponding Author: & \multicolumn{2}{|l|}{$\begin{array}{l}\text { Emilia Fushimi, Eng. } \\
\text { Instituto LEICI, UNLP-CONICET } \\
\text { La Plata, Buenos Aires ARGENTINA }\end{array}$} \\
\hline \multicolumn{3}{|l|}{$\begin{array}{l}\text { Corresponding Author Secondary } \\
\text { Information: }\end{array}$} \\
\hline Corresponding Author's Institution: & \multicolumn{2}{|l|}{ Instituto LEICI, UNLP-CONICET } \\
\hline \multicolumn{3}{|l|}{$\begin{array}{l}\text { Corresponding Author's Secondary } \\
\text { Institution: }\end{array}$} \\
\hline First Author: & \multicolumn{2}{|l|}{ Emilia Fushimi, Eng. } \\
\hline \multicolumn{3}{|l|}{ First Author Secondary Information: } \\
\hline \multirow[t]{4}{*}{ Order of Authors: } & \multicolumn{2}{|l|}{ Emilia Fushimi, Eng. } \\
\hline & \multicolumn{2}{|l|}{ María Cecilia Serafini, Eng. } \\
\hline & \multicolumn{2}{|l|}{ Hernán De Battista, Phd. } \\
\hline & \multicolumn{2}{|l|}{ Fabricio Garelli, Phd. } \\
\hline \multicolumn{3}{|c|}{ Order of Authors Secondary Information: } \\
\hline \multirow[t]{3}{*}{ Funding Information: } & $\begin{array}{l}\text { Universidad Nacional de La Plata } \\
(11 / 1216)\end{array}$ & Not applicable \\
\hline & $\begin{array}{l}\text { Consejo Nacional de Investigaciones } \\
\text { Científicas y Técnicas } \\
\text { (PIP 112-201501-00837) }\end{array}$ & Mr. Fabricio Garelli \\
\hline & $\begin{array}{l}\text { Agencia Nacional de Promoción Científica } \\
\text { y Tecnológica } \\
\text { (PICT2017-3211) }\end{array}$ & Mr. Hernán De Battista \\
\hline Abstract: & \multicolumn{2}{|c|}{$\begin{array}{l}\text { Artificial pancreas (AP) systems have shown to improve glucose regulation in type } 1 \\
\text { diabetes (T1D) patients. However, full closed-loop performance remains a challenge } \\
\text { particularly in children and adolescents, since these age groups often present the } \\
\text { worst glycemic control. In this work, an algorithm based on switched control and time- } \\
\text { varying insulin-on-board (IOB) constraints is presented. The proposed control strategy } \\
\text { is evaluated in silico using the FDA-approved UVA/Padova simulator and its } \\
\text { performance contrasted with the previously introduced } \\
\text { Automatic Regulation of Glucose (ARG) algorithm in the pediatric population. The effect } \\
\text { of unannounced meals is also explored. Results indicate that the proposed strategy } \\
\text { achieves lower hypo- and hyperglycemia than the ARG for both announced and } \\
\text { unannounced meals. }\end{array}$} \\
\hline \multirow[t]{2}{*}{ Suggested Reviewers: } & \multicolumn{2}{|c|}{$\begin{array}{l}\text { Fabián León-Vargas } \\
\text { Universidad Antonio Nariño } \\
\text { fabianleon@uan.edu.co } \\
\text { Expertise Artificial Pancreas control algorithms involving constrained control }\end{array}$} \\
\hline & \multicolumn{2}{|l|}{$\begin{array}{l}\text { Arthur Bertachi } \\
\text { Universitat de Girona } \\
\text { arthurbertachi@gmail.com }\end{array}$} \\
\hline
\end{tabular}


Expertise in the Artificial Pancreas

Patricio Colmegna

University of Virginia

pc2jx@virginia.edu

Expertise in Artificial Pancreas control algorithms based on switched control and IOB constraints

José García-Tirado

University of Virginia

jg2bt@virginia.edu

Expertise in Artificial Pancreas control algorithms

Pablo Rivadeneira

Universidad Nacional de Colombia

psrivade@unal.edu.co

Expertise in the Artificial Pancreas. 
Automatic glycemic regulation for the pediatric population based on switched control and timevarying IOB constraints: an in silico study

Emilia Fushimi, Eng ${ }^{1,2}$, María Cecilia Serafini, Eng ${ }^{1,3}$, Hernán De Battista, Dr. Eng ${ }^{1,2}$, Fabricio Garelli, Dr.

$$
\text { Eng }^{1,2}
$$

Author Affiliations: ${ }^{1}$ Universidad Nacional de La Plata (UNLP), ${ }^{2}$ National Scientific and Technical Research Council (CONICET), ${ }^{3}$ Comisión de Investigaciones Científicas de la Provincia de Buenos Aires (CICpBA).

Emilia Fushimi

Grupo de Control Aplicado (GCA)

Instituto LEICI (UNLP-CONICET),

Facultad de Ingeniería,

Universidad Nacional de La Plata (UNLP)

Calle 48 no162 esq 117

La Plata, Argentina (1900)

$+542214259306$

emilia.fushimi@ing.unlp.edu.ar

Hernán De Battista

Grupo de Control Aplicado (GCA)

Instituto LEICI (UNLP-CONICET),

Facultad de Ingeniería,

Universidad Nacional de La Plata (UNLP)

Calle 48 no162 esq 117
María Cecilia Serafini

Grupo de Control Aplicado (GCA)

Instituto LEICI (UNLP-CONICET),

Facultad de Ingeniería,

Universidad Nacional de La Plata (UNLP)

CICpBA.

Calle 48 no162 esq 117

La Plata, Argentina (1900)

$+542214259306$

\section{cecilia.serafini@ing.unlp.edu.ar}

Fabricio Garelli

Grupo de Control Aplicado (GCA)

Instituto LEICI (UNLP-CONICET),

Facultad de Ingeniería,

Universidad Nacional de La Plata (UNLP)

Calle 48 no162 esq 117 
La Plata, Argentina (1900)

$+542214259306$

deba@ing.unlp.edu.ar
La Plata, Argentina (1900)

$+542214259306$

fabricio@ing.unlp.edu.ar

Corresponding author: Emilia Fushimi. National Scientific and Technical Research Council (CONICET). Universidad Nacional de La Plata (UNLP), Facultad de Ingeniería, Instituto LEICl (Grupo de Control Aplicado), Calle 47 no162 esq 117, La Plata, Argentina (1900). E-mail: emilia.fushimi@ing.unlp.edu.ar

Abstract: Artificial pancreas (AP) systems have shown to improve glucose regulation in type 1 diabetes (T1D) patients. However, full closed-loop performance remains a challenge particularly in children and adolescents, since these age groups often present the worst glycemic control. In this work, an algorithm based on switched control and time-varying insulin-on-board (IOB) constraints is presented. The proposed control strategy is evaluated in silico using the FDA-approved UVA/ Padova simulator and its performance contrasted with the previously introduced Automatic Regulation of Glucose (ARG) algorithm in the pediatric population. The effect of unannounced meals is also explored. Results indicate that the proposed strategy achieves lower hypo- and hyperglycemia than the ARG for both announced and unannounced meals.

Keywords: Artificial pancreas, Switched control, Insulin on board, Constrained control.

Acknowledgements: This research has been supported by the Argentinean Government (PICT 20173211 Agencia Nacional de Promoción Científica y Tecnológica, PIP 112-201501-00837 CONICET, UNLP 11/I216).

Conflict-of-Interest Disclosure: None.

Figure and Table counting: 8 Figures and 12 Tables.

Total number of words of the manuscript: 6516 
Number of words of the abstract: 114

\section{Authors Short Bio:}

Emilia Fushimi: Born in Buenos Aires, Argentina, in 1993. Received the B.S.E.E. degree from the National University of La Plata (UNLP), La Plata, Argentina, in 2016, where she is currently working toward the Ph.D. degree in the Laboratory of Industrial Electronics, Control and Instrumentation. She is also a graduate student assistant in the Department of Electrical Engineering, UNLP.

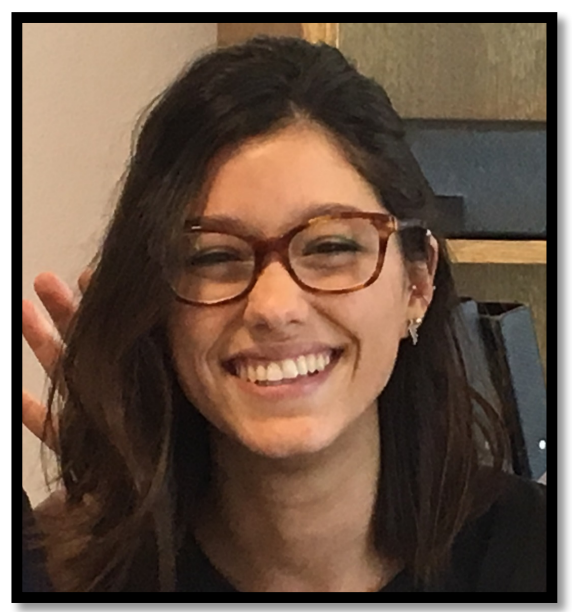

María Cecilia Serafini: Born in Bahía Blanca, Argentina, in 1986. She got her B.S.E.E. from the National University of The South (UNS, Bahía Blanca) in 2018 and is currently working towards her Ph.D. degree in the Laboratory of Industrial Electronics, Control and Instrumentation (LEICI) at the National University of La Plata (UNLP). 


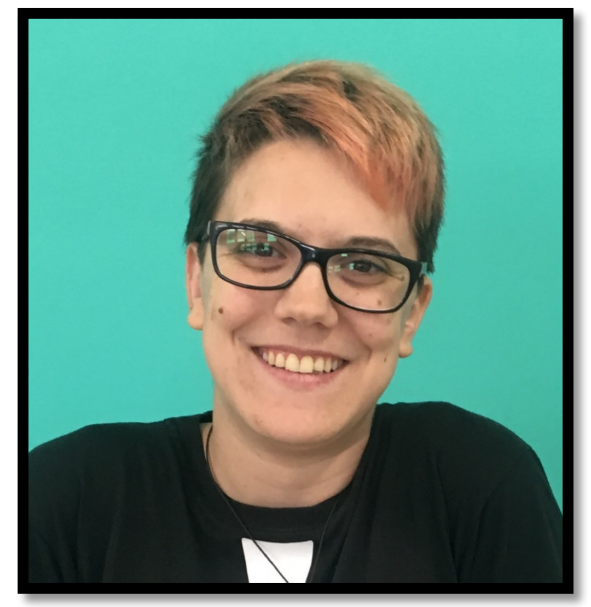

Hernán De Battista: Born in La Plata, Argentina, in 1968. He received the M.S.Eng. degree with highest honors in 1994 and the PhD degree in 2000, both from La Plata National University, Argentina. Currently he is Full Professor at the Faculty of Engineering of La Plata National University and Principal Researcher of the National Research Council of Argentina (CONICET). His main research interests are in nonlinear control and its applications to renewable energies and biological systems. HDB received the E. Galloni award from the Argentine Academy of Exact, Physical and Natural Sciences in 2002 and the S. Gershanik award from the Buenos Aires Academy of Engineering in 2006.

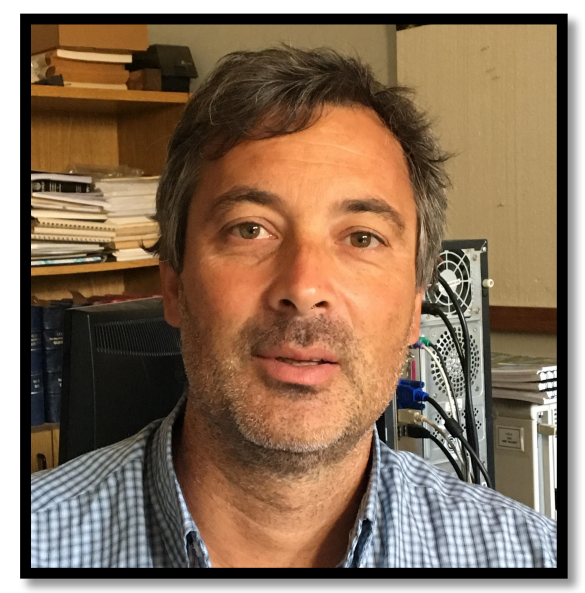

Fabricio Garelli: Fabricio Garelli is Full Professor at National University of La Plata (UNLP) and Official Member of the National Research Council of Argentina (CONICET). He is the author of an awarded Ph.D. 
Thesis, an IET book and more than a hundred of journal or conference papers. His research work focuses on multivariable constrained control and estimation via sliding mode techniques, with application to industrial processes/bioprocesses, robotics and biomedical engineering (artificial pancreas).

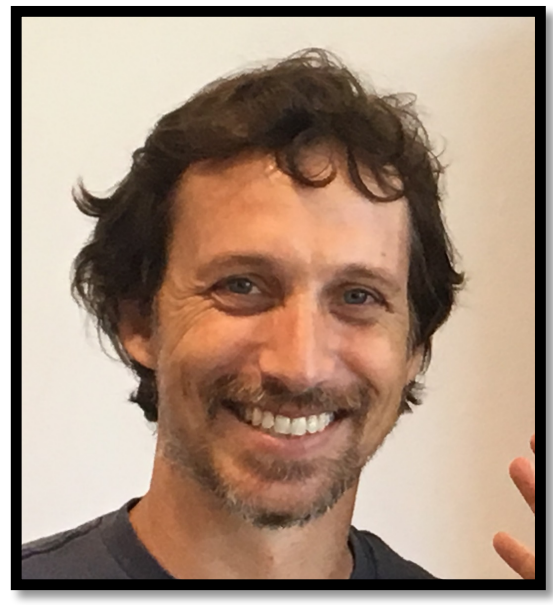




\title{
Automatic glycemic regulation for the pediatric population based on switched control and time-varying IOB constraints: an in silico study
}

\author{
E. Fushimi - M. C. Serafini - H. De \\ Battista - F. Garelli
}

Received: date / Accepted: date

\begin{abstract}
Artificial pancreas (AP) systems have shown to improve glucose regulation in type 1 diabetes (T1D) patients. However, full closed-loop performance remains a challenge particularly in children and adolescents, since these age groups often present the worst glycemic control. In this work, an algorithm based on switched control and time-varying insulin-on-board (IOB) constraints is presented. The proposed control strategy is evaluated in silico using the FDA-approved UVA/ Padova simulator and its performance contrasted with the previously introduced Automatic Regulation of Glucose (ARG) algorithm in the pediatric population. The effect of unannounced meals is also explored. Results indicate that the proposed strategy achieves lower hypo- and hyperglycemia than the ARG for both announced and unannounced meals.
\end{abstract}

Keywords Artificial pancreas · Switched control · Insulin on board · Constrained control

\section{Introduction}

Artificial pancreas (AP) systems consist of a subcutaneous insulin pump connected to a continuous glucose monitoring (CGM) sensor through a control algorithm that automatically calculates insulin doses according to CGM measures [1]. Unfortunately, the subcutaneous route introduces considerable issues, including large delays in glucose measurement and insulin action [2].

The vast majority of AP systems are based on model predictive control (MPC) [3-5] , proportional-integral-derivative (PID) [6-8] and fuzzy logic (FL) [9] (see [10] for a thorough description of the current situation). Since high model uncertainty and large delays limit the autonomy of the glucose controller, most of these control

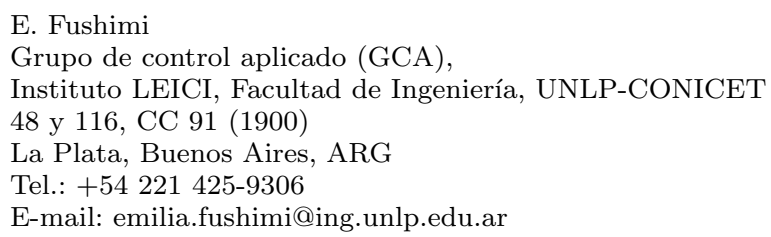


systems are hybrid, i.e., a combination of manual meal boluses and a control algorithm that adjusts basal infusion. However, carbohydrates (CHO) counting implies an important burden and risk for people with diabetes [11].

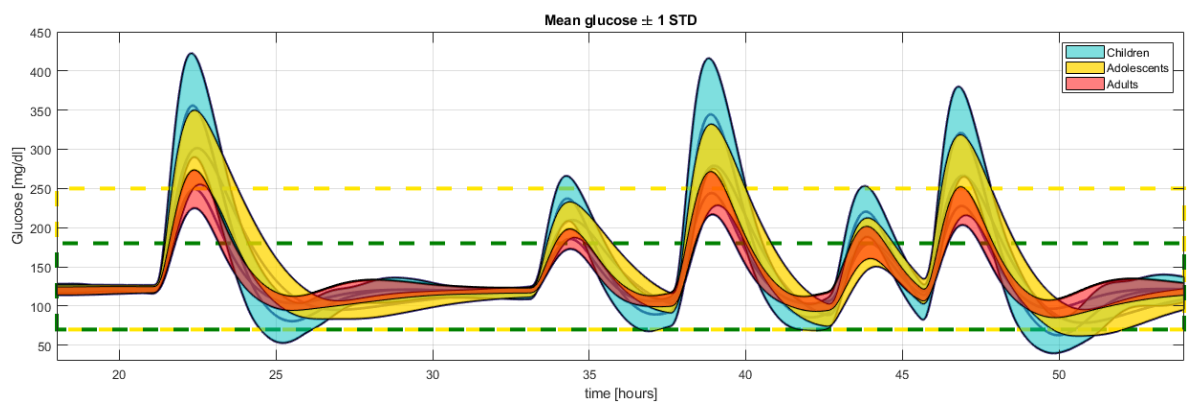

Fig. 1 Glycemia (mean \pm 1 std) vs time of the three age ranges available in the UVA/Padova simulator when facing 5 meals during 36 hs using the ARG controller (10 adults - red, 10 adolescents - yellow, 10 children - cyan). Dashed green lines delimit the desired range ([70-180] $\mathrm{mg} / \mathrm{dl})$ and the yellow show the acceptable range $([70-250] \mathrm{mg} / \mathrm{dl})$.

Recently, a control algorithm without pre-meal insulin boluses called Automatic Regulation of Glucose (ARG) was proposed and clinically evaluated in adult subjects with type 1 diabetes (T1D) at the Hospital Italiano de Buenos Aires (HIBA) [12,13]. This algorithm consists of an inner switched linear quadratic gaussian (SLQG) controller and an outer sliding mode safety layer called Safety Auxiliary Feedback Element (SAFE) [14]. The inner controller switches between an aggressive LQG controller to compensate for the effect of meals and other large perturbations, and a conservative LQG controller to maintain euglycemia at all other times [13]. A meal announcement is required at meal times to trigger a listening mode in which the controller waits to detect an increasing trend on CGM readings to switch to the aggressive controller. Although promising results were obtained, more intensive trials are necessary to assess the controller performance. Particularly, trials involving children and adolescents are fundamental since these age groups often present poor glycemic control and respond to treatment significantly different from adults. To this end, a clinical trial in collaboration with the pediatric hospital Garrahan in Buenos Aires is expected to take place in 2020.

Figure 1 shows the response of the different age groups available in the UVA/Padova simulator to a 5 meal scenario similar to the one carried out at the HIBA using the ARG algorithm. It can be seen that children and adolescents present larger glucose excursions and more pronounced hypo- and hyperglycemic episodes. Therefore, it is reasonable to consider an enhanced control approach in order to control the pediatric population.

Additionally, the pediatric population presents other obstacles concerning glycemic control. Children and adolescents are more prone to forget to announce meals and to suffer from diabetic burnout [15]. Therefore, an unannounced meal strategy would particularly benefit this age group. AP systems that do not require any kind of meal announcement involving both single-hormone [16,17] and dual-hormone [18] therapy have been proposed and tested in the adult population. Particularly, an in silico study evaluating the performance of the ARG algorithm with unan- 
nounced meals was made [19]. This work introduced an automatic switching signal generator (SSG), which commanded the switching between aggressive and conservative controllers, thus eliminating the listening mode. Even though encouraging results were achieved, the study was only a preliminary evaluation considering the adult population. To the extent of the authors knowledge, no single-hormone full closed-loop AP system has been tested in children or adolescents.

In this paper, a further step on the ARG algorithm is given, aiming to be tested in vivo in the Garrahan clinical trials. The proposed controller takes advantage of the ARG algorithm switched strategy (SLQG), while introducing a redesigned switching logic and time-varying IOB constraints. This kind of constraint is called Amplitude Enable (AE) and allows the controller to act more aggressively at the beginning of the meal without risking postprandial hypoglycemia [20]. The proposed control strategy $\left(A R G_{A E}\right)$ is then evaluated in silico using the FDA-approved UVA/Padova simulator and contrasted to the ARG algorithm. Simulation scenarios involving unannounced meals are also explored using the SSG mentioned above.

\section{Materials and Methods}

In this section, the ARG algorithm, the automatic SSG and the proposed $A R G_{A E}$ are described.

\subsection{The ARG Algorithm}

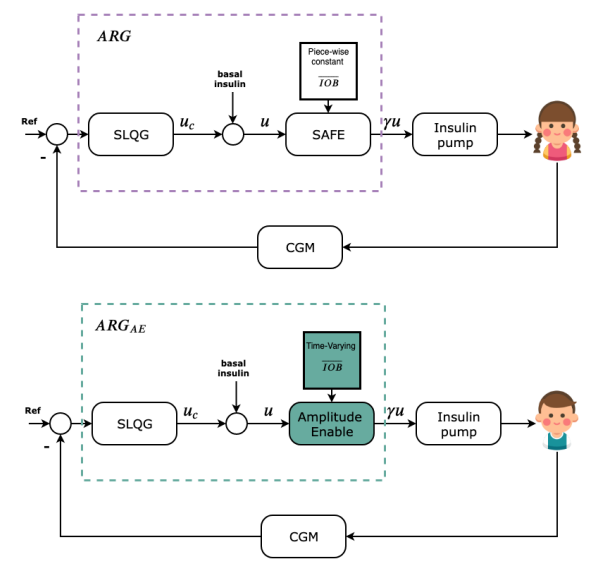

Fig. 2 Block diagram of the $A R G$ and $A R G_{A E}$ algorithms

The ARG algorithm regulates glycemia without delivering open-loop prandial boluses. Figure 2 shows a block diagram of the ARG algorithm and the $A R G_{A E}$ algorithm, highlighting with fill boxes the new components of the latter. The main controller is an SLQG, which switches between an aggressive controller $K_{2}$, which is in charge of compensating for the effects of the meals, and a conservative 
controller $K_{1}$, which maintains glucose levels in the desired range during fasting periods [13]. The output of this block is the insulin dose calculated by the SLQG $\left(u_{c}\right)$.

Since the controller does not have integral action, the open-loop basal insulin is added to $u_{c}$, yielding $u$. The signal $u$ would command the insulin pump if the SAFE layer was not present. However, the SAFE shapes $u$ through the signal $\gamma$ $(\gamma \in[0,1])$ in order to avoid violating an imposed restriction on the insulin-onboard (IOB). Therefore, the insulin dose that is finally delivered to the patient is $\gamma u$.

For the HIBA clinical trials, the switching from $K_{1}$ to $K_{2}$ was made using a meal announcement. The announcement triggered a listening mode where the algorithm waited to detect an increasing trend on CGM readings to switch to $K_{2}$. It is worth highlighting that the meal announcement was not used to deliver meal priming boluses and no $\mathrm{CHO}$ counting was required. Instead, the user informed the size of the meal using one of three categories: small, medium and large. This information was used to tune the SAFE layer (see equation (1) below). On the other hand, the switching form $K_{2}$ to $K_{1}$ occurred automatically after one hour.

The SAFE layer in the ARG algorithm uses piecewise constant IOB constraints depending on the announced meal classification, according to the following equations:

- Small meals < 35 gCHO:

$\overline{I O B}=\mathrm{IOB}_{\mathrm{ss}}+40 \mathrm{gCHO} / \mathrm{CR}$,

- Medium meals $[35,65)$ gCHO:

$\overline{I O B}=\mathrm{IOB}_{\mathrm{ss}}+55 \mathrm{gCHO} / \mathrm{CR}$,

- Large meals $\geq 65$ gCHO:

$\overline{I O B}=\mathrm{IOB}_{\mathrm{ss}}+70 \mathrm{gCHO} / \mathrm{CR}$,

where $\mathrm{IOB}_{\mathrm{ss}}$ is the IOB with the basal insulin infusion, and CR is each patients carbohydrate ratio.

The ARG algorithm has been validated in vivo in adult patients at the HIBA clinical trial. Although results were encouraging, in silico evaluation showed that the performance worsened when controlling the pediatric population. As shown in figure 1, hyper- and hypoglycemia are more prominent for children and adolescents than for adults.

2.2 Switching Signal Generator (SSG)

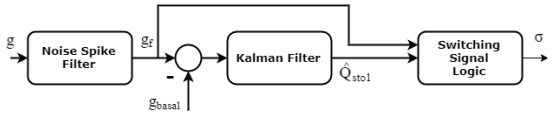

Fig. 3 Block diagram of the meal detection algorithm.

The signal that commands when the ARG algorithm is triggered into its aggressive mode is $\sigma$. In order to eliminate the need for announcing the meal manually, a meal detection algorithm to establish $\sigma$ based on CGM readings was proposed in $[19]$. 
Figure 3 shows a block diagram of the proposed meal detector. The first block is a noise spike filter (NSF) that limits the maximum BG rate of change to 3 $\mathrm{mg} / \mathrm{dl} / \mathrm{min}[21]$. The filtered signal $g_{f}$ is the input to the second block, which is a Kalman filter. The Kalman filter is used to estimate the amount of glucose in the first phase of the stomach $\hat{Q}_{s t o 1}[22]$. The Switching Signal block determines whether a meal is present or not using predefined thresholds for $g_{f}$ and $\hat{Q}_{s t o 1}$. See [19] for further details on the SSG.

\subsection{The ARG Algorithm with Amplitude Enable $\left(A R G_{A E}\right)$}

According to [23], the theoretically optimal treatment for glucose regulation is the basal-superbolus open-loop therapy. However, the high uncertainty present in meal size and composition, patient parameters, and sensor readings make closed-loop control highly convenient. With this in mind, an IOB limitation strategy based on open-loop therapy was introduced, called the AE [20].

The AE block uses sliding mode control in order to avoid the violation of an imposed restriction. In this case, the restriction is time-varying, shaped as the open-loop therapy IOB profile multiplied by a constant $\beta>1$, in order to give the controller an extra degree of freedom. Figure 4 shows the IOB constraint for different values of $\beta$.

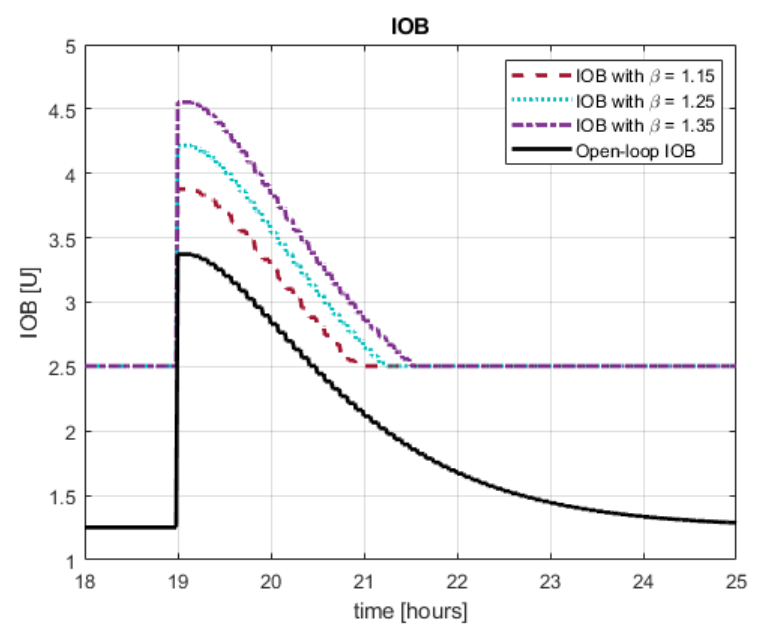

Fig. $4 \overline{I O B}$ for different values of $\beta$

The AE layer, can work around any main controller. Here, it is combined with a switched strategy (the ARG algorithm SLQG), since using a very aggressive controller at all times might generate undesired oscillations, and a conservative one might not be strong enough to compensate large perturbations like meal intake.

In order to further reduce hyperglycemia risk, $\overline{I O B}$ is set to never be less than two times $\mathrm{IOB}_{\mathrm{ss}}$. Additionally, the switching policy between $K_{1}$ and $K_{2}$ is redesigned: the commutation from conservative to aggressive is made immediately 
after a meal is announced or detected. Then, the controller switches from aggressive to conservative only when a decreasing trend (three consecutive samples) in CGM readings is observed.

When meals are announced, meal classification is used in order to compute the $\overline{I O B}$. When meals are automatically detected, a medium-sized meal is assumed.

An illustrative example of the ARG and the $A R G_{A E}$ insulin infusion and IOB profiles when facing a meal is shown in figure 5. Since for the SAFE layer $\overline{I O B}$ is a constant piecewise function, insulin delivery is lower than with the $A R G_{A E}$ at meal intake, and close to basal values in the postprandial period. On the other hand, it can be seen that the insulin response generated by the $A R G_{A E}$ is similar to a superbolus: large insulin spikes at meal time, followed by a cut in basal insulin delivery. The shape of the IOB constraint used for the AE has the advantage of allowing large insulin doses at the beginning of the meal, and reducing them gradually as the meal effect ends. The AE allows this non-linear behaviour to an otherwise linear controller like the LQG, which is a distinctive feature of the proposal.
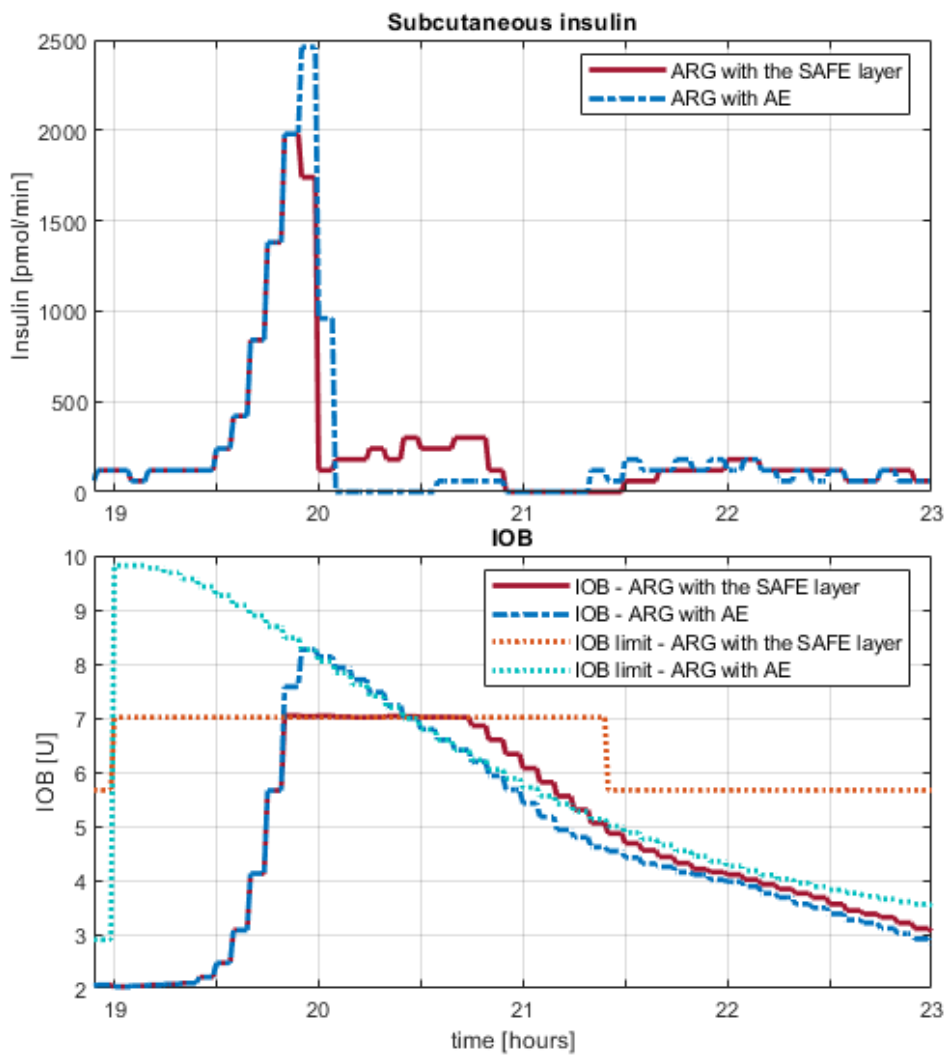

Fig. 5 Example of the $\overline{I O B}$, IOB and resulting insulin dose for the ARG with the SAFE layer (red) and for the $A R G_{A E}$ (blue). 


\section{Results}

In this section, results from in silico tests are shown. First, the ARG with the amplitude enable layer $\left(A R G_{A E}\right)$ is evaluated considering a medium-sized meal for different values of $\beta$ using the adolescent and children age groups available in the UVA/Padova simulator. The results are compared to the ARG and a retuned ARG $\left(A R G_{80 \%}\right)$, in which the $\overline{I O B}$ is reduced to $\% 80$ of the value obtained using (1) in order to reduce frequency of hypoglycemia episodes. The meal is announced at the time of intake, with the appropriate meal classification (medium).

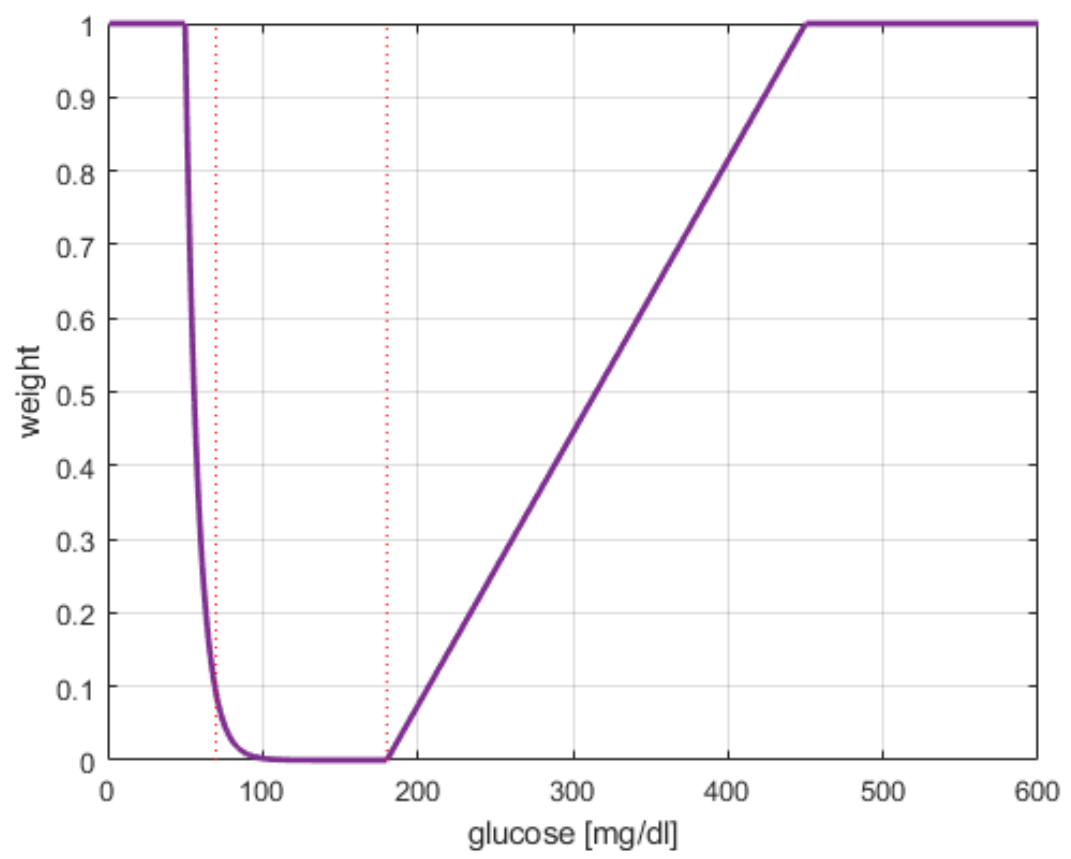

Fig. 6 Penalty index: weight vs. glucose concentration

Then, according to the first set of simulations, an adequate value for $\beta$ is selected. To this end, a performance index based on hyper- and hypoglycemia risk is used $[3,24]$. Figure 6 shows the weigth vs. glycemia used to compute this index. As it can be observed, this index penalizes values outside the desired range. Hypoglycemia is more heavily penalized as it can present the most dangerous consequences. It can be seen from the function shape that a low index is desired.

For the second set of simulations, meal size error is considered using a small and a large-sized meal while announcing a medium meal.

Lastly, the same scenarios are simulated without the use of meal announcement. For all the different ARG configurations, a medium size meal is assumed whenever a meal is detected. 
For all simulations, meal intake takes place at $t=1$ and is followed by a 9 hour post-prandial observation period. CGM noise is also considered using a DEXCOM CGM model.

3.1 in silico evaluation with announced meals

Here, the results from the simulation sets using meal announcement are shown and analyzed.

3.1.1 Medium-sized meal - $\beta$ sweep

Table 1 Results (\% of time) for the 10 adolescents of the UVA/Padova Simulator using the $A R G, A R G_{80 \%}$ and $A R G_{A E}$ with different values of $\beta$. Meal size: 55 gCHO (medium). Announced meal size: Medium.

\begin{tabular}{llllll}
\hline $\begin{array}{l}\text { Adolescents } \\
\text { Announced 55g Meal }\end{array}$ & \multirow{2}{*}{$A R G$} & \multirow{2}{*}{$A R G_{80 \%}$} & $\begin{array}{l}A R G_{A E} \\
\beta=1.35\end{array}$ & $\begin{array}{l}A R G_{A E} \\
\beta=1.40\end{array}$ & $\begin{array}{l}A R G_{A E} \\
\beta=1.45\end{array}$ \\
\hline Hyperglycemia & $23.1+-4.2$ & $27.2+-4.8$ & $22.5+-4.4$ & $22.0+-4.3$ & $21.5+-4.3$ \\
Hypoglycemia & $1.0+-3.2$ & $0.0+-0.0$ & $0.0+-0.0$ & $0.0+-0.0$ & $0.5+-1.7$ \\
Severe hyperglycemia & $11.7+-5.8$ & $13.7+-6.0$ & $10.7+-6.5$ & $10.5+-6.4$ & $10.3+-6.3$ \\
Desired range & $75.9+-6.5$ & $72.8+-4.8$ & $77.5+-4.4$ & $78.0+-4.3$ & $77.9+-4.4$ \\
Acceptable range & $87.3+-7.8$ & $86.3+-6.0$ & $89.3+-6.5$ & $89.5+-6.4$ & $89.2+-6.9$ \\
\hline Penalty Index & 7.13 & 7.70 & 6.51 & 6.50 & 6.65
\end{tabular}

Table 1 shows the mean \pm 1 std of the $\%$ time in hyperglycemia $(>180 \mathrm{mg} / \mathrm{dl})$, hypoglycemia $(<70 \mathrm{mg} / \mathrm{dl})$, severe hyperglycemia $(>250 \mathrm{mg} / \mathrm{dl})$, the desired range $(70-180 \mathrm{mg} / \mathrm{dl})$ and the acceptable range $(70-250 \mathrm{mg} / \mathrm{dl})$ for the 10 adolescents available in commercial version of the UVA/Padova simulator using the $A R G$, the $A R G_{80 \%}$ and the $A R G_{A E}$ for a 55 gCHO meal. It can be observed that while the $A R G_{80 \%}$ reduces hypoglycemia, it significantly increases time spent in hyperglycemia, thus achieving a higher penalty index than the ARG. On the other hand, $A R G_{A E}$ achieves the same reduction of hypoglycemia episodes, while also diminishing hyperglycemia when an adequate $\beta$ is selected. In this case, a $\beta=1.4$ achieves the most time in the desired and acceptable ranges and the lowest penalty index (grey column).

Figure 7 shows the glucose, insulin infusion, controller mode and IOB over time for the $A R G$ and the $A R G_{A E}$ with $\beta=1.4$ for adolescent \#004 of the UVA/Padova simulator. As expected, the $A R G_{A E}$ administers larger doses of insulin at the beginning of the meal and cuts insulin afterwards, slowly returning to basal delivery when $\mathrm{BG}$ values start to decrease. On the other hand, the constant $\overline{I O B}$ in the $A R G$ results in a more distributed insulin dosage by the end of the meal. This causes a larger hyperglycemia peak with a higher risk of postprandial hypoglycemia. Thus, it can be observed that the $A R G_{A E}$ generates a slightly lower peak in BG than the $A R G$, while achieving safer postprandial $B G$ values.

Table 2 shows the same metrics as table 1 but for the 10 children available in the UVA/Padova simulator, also for the $A R G$, the $A R G_{80 \%}$ and the $A R G_{A E}$ with different values of $\beta$. In this case, the $A R G_{A E}$ and the $A R G_{80 \%}$ show significant reduction in the time in hypoglycemia compared to the ARG. However, $A R G_{80 \%}$ 

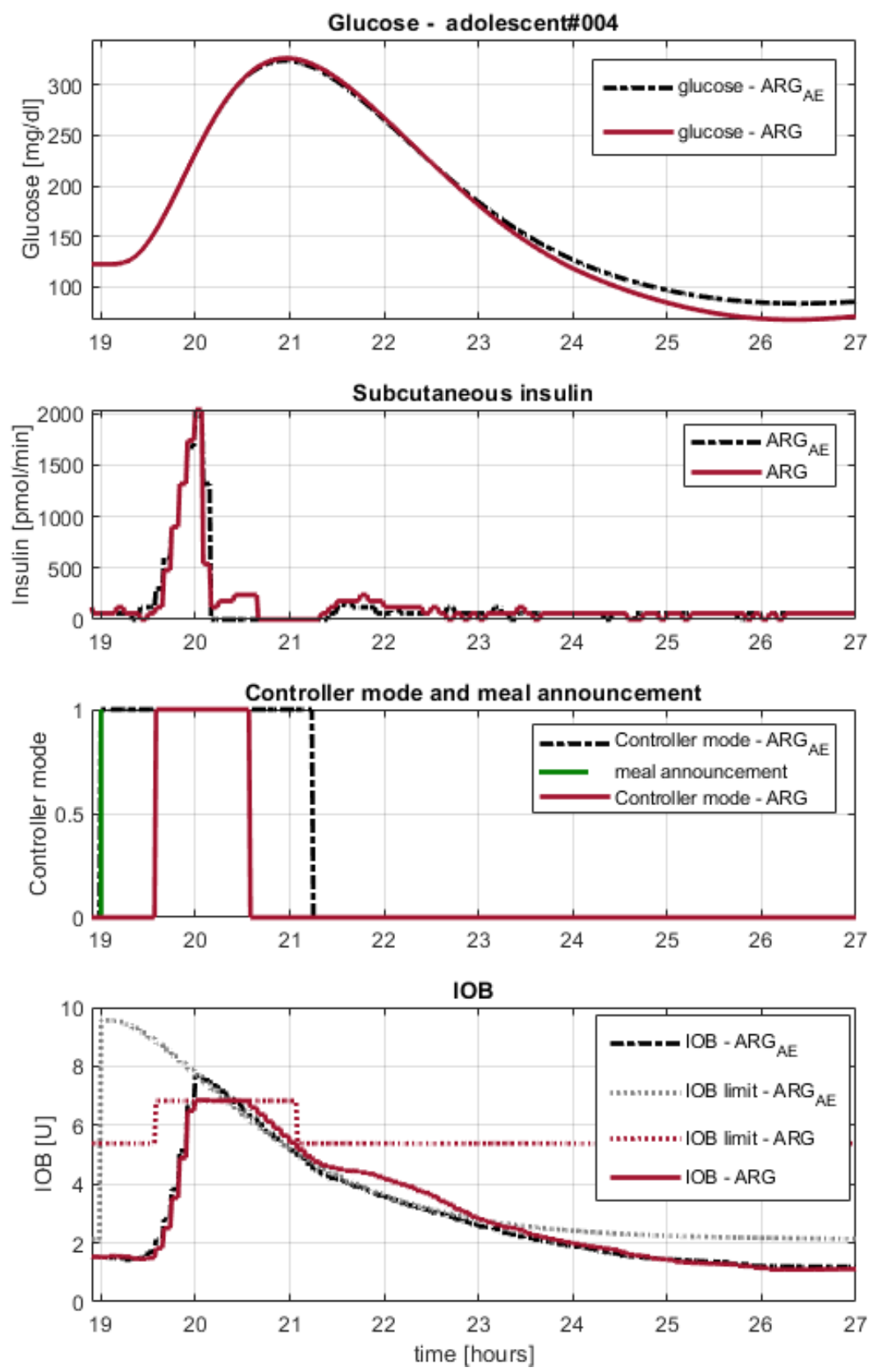

Fig. 7 Glucose, insulin, controller mode (1 - aggressive, 0 - conservative) and IOB evolution over time of adolescent 4 of the UVA/Padova simulator using the $A R G$ (solid red) and the $A R G_{A E}$ with $\beta=1.4$ (dashed-dotted black).

shows a pronounced increase in hyperglycemia. Additionally, time in range is significantly improved by the $A R G_{A E}$. 
Table 2 Results (\% of time) for the 10 children of the UVA/Padova Simulator using the $A R G$, $A R G_{80 \%}$ and $A R G_{A E}$ with different values of $\beta$. Meal size: 55 gCHO (medium). Announced meal size: Medium.

\begin{tabular}{llllll}
\hline $\begin{array}{l}\text { Children } \\
\text { Announced 55g Meal }\end{array}$ & $A R G$ & $A R G_{80 \%}$ & $\begin{array}{l}A R G_{A E} \\
\beta=1.20\end{array}$ & $\begin{array}{l}A R G_{A E} \\
\beta=1.25\end{array}$ & $\begin{array}{l}A R G_{A E} \\
\beta=1.30\end{array}$ \\
\hline Hyperglycemia & $19.9+-3.3$ & $24.2+-4.8$ & $21.1+-4.4$ & $20.6+-4.2$ & $19.7+-3.8$ \\
Hypoglycemia & $6.0+-8.8$ & $0.0+-0.0$ & $0.5+-1.7$ & $1.5+-3.3$ & $3.1+-5.7$ \\
Severe hyperglycemia & $12.8+-4.5$ & $15.7+-4.7$ & $12.6+-5.3$ & $12.3+-5.2$ & $11.9+-5.0$ \\
$\begin{array}{l}\text { Desired range } \\
\text { Acceptable range }\end{array}$ & $74.0+-7.1$ & $75.8+-4.8$ & $78.3+-4.7$ & $77.9+-4.6$ & $77.2+-5.4$ \\
\hline Penalty Index & $81.1+-8.3$ & $84.3+-4.7$ & $86.9+-6.0$ & $86.2+-6.6$ & $85.1+-7.9$ \\
\hline
\end{tabular}

The results for both children and adolescents using the $A R G_{80 \%}$ show that even though hypoglycemia can be reduced, it is in exchange of a significant increase in hyperglycemia and severe hyperglycemia.

\subsubsection{Overestimated announced meal}

Here, a $40 \mathrm{gCHO}$ (small) meal is considered but a medium meal is announced in order to evaluate the algorithms performance when meals are overestimated.

Table 3 Results (\% of time) for the 10 adolescents of the UVA/Padova Simulator using the $A R G$ and $A R G_{A E}$ with $\beta=1.4$. Meal size: $40 \mathrm{gCHO}$ (small). Announced meal size: Medium.

\begin{tabular}{lll}
\hline $\begin{array}{l}\text { Adolescents } \\
\text { Overestimated Meal }\end{array}$ & $A R G$ & $A R G_{A E}$ \\
\hline Hyperglycemia & $17.7+-4.2$ & $16.7+-4.3$ \\
Hypoglycemia & $4.4+-9.3$ & $3.5+-6.2$ \\
Severe hyperglycemia & $4.1+-5.6$ & $3.8+-5.3$ \\
Desired range & $77.9+-10.6$ & $79.8+-6.6$ \\
Acceptable range & $91.5+-10.6$ & $92.7+-7.7$ \\
\hline Penalty Index & 5.62 & 4.23
\end{tabular}

Table 3 shows the same metrics as table 1 for the 10 adolescents available in the UVA/Padova simulator using the $A R G$ and the $A R G_{A E}$ with $\beta=1.4$. Since the meal is overestimated, the time spent in hyperglycemia is lower for all control strategies compared with the 55 gCHO meal. Also, hypoglycemia is increased for the $A R G$. On the other hand, hypoglycemia is reduced with the $A R G_{A E}$, while slightly lowering time in hyperglycemia. If a more conservative $\beta$ had been chosen in order to prioritize hypoglycemia prevention, hypoglycemia can be further reduced using the $A R G_{A E}$, For example, with $\beta=1.35$ : \% time in hyperglycemia $=17.0 \pm 4.3$, hypoglycemia $=1.4 \pm 3.6$, severe hyperglycemia $=$ $3.8 \pm 5.4$, the desired range $=81.6 \pm 5.1$ and the acceptable range $=94.8 \pm 6.0$, yielding an index of 3.76 .

Table 4 shows the same metrics as table 3 but for the 10 children available in the UVA/Padova simulator, also for the $A R G$ and the $A R G_{A E}$ with $\beta=1.25$ based on the results form 3.1.1. In this case, the $A R G$ and the $A R G_{A E}$ show the same $\%$ of time in hyperlgycemia, while obtaining similar hypoglycemia. However, like 
Table 4 Results (\% of time) for the 10 children of the UVA/Padova Simulator using the $A R G$ and $A R G_{A E}$ with $\beta=1.25$. Meal size: $40 \mathrm{gCHO}$ (small). Announced meal size: Medium.

\begin{tabular}{|c|c|c|}
\hline $\begin{array}{l}\text { Children } \\
\text { Overestimated Meal }\end{array}$ & $A R G$ & $A R G_{A E}$ \\
\hline Hyperglycemia & $15.7+-3.2$ & $15.2+-3.4$ \\
\hline Hypoglycemia & $6.8+-9.0$ & $7.2+-10.2$ \\
\hline Severe hyperglycemia & $7.4+-3.9$ & $6.4+-4.0$ \\
\hline Desired range & $77.6+-7.1$ & $77.6+-8.0$ \\
\hline Acceptable range & $85.9+-8.8$ & $86.4+-10.1$ \\
\hline Penalty Index & 9.39 & 7.95 \\
\hline
\end{tabular}

with the adolescent age group, if a more restrictive $\beta$ were selected, the $A R G_{A E}$ has the ability of reducing hypoglycemia while maintaining low hyperglycemia. For example, with $\beta=1.15: \%$ time in hyperglycemia $=16.2 \pm 3.7$, hypoglycemia $=2.9 \pm 4.4$, severe hyperglycemia $=6.9 \pm 4.1$, the desired range $=80.9 \pm 2.6$ and the acceptable range $=90.2 \pm 4.8$, yielding an index of 5.24

\subsubsection{Underestimated announced meal}

Here, a $70 \mathrm{gCHO}$ (large) meal is considered but a medium meal is announced in order to evaluate the algorithms performance when meals are underestimated.

Table 5 Results (\% of time) for the 10 adolescents of the UVA/Padova Simulator using the $A R G$ and $A R G_{A E}$ with $\beta=1.4$. Meal size: 70 gCHO (large). Announced meal size: Medium.

\begin{tabular}{lll}
\hline $\begin{array}{l}\text { Adolescents } \\
\text { Underestimated Meal }\end{array}$ & $A R G$ & $A R G_{A E}$ \\
\hline Hyperglycemia & $27.8+-4.2$ & $26.5+-4.5$ \\
Hypoglycemia & $0.0+-0.0$ & $0.0+-0.0$ \\
Severe hyperglycemia & $17.9+-4.6$ & $16.8+-4.8$ \\
Desired range & $72.2+-4.2$ & $73.5+-4.5$ \\
Acceptable range & $82.1+-4.6$ & $83.2+-4.8$ \\
\hline Penalty Index & 10.94 & 10.15
\end{tabular}

Table 5 shows the same metrics as table 1 for the 10 adolescents available in the UVA/Padova simulator using the $A R G$ and the $A R G_{A E}$ with $\beta=1.4$. Since the meal is underestimated, the time spent in hyperglycemia is higher for both control strategies, and hypoglycemia is avoided. On the other hand, hyperglycemia and severe hyperglycemia are slightly improved with the $A R G_{A E}$, thus achieving the lowest index.

Table 6 shows the same metrics as table 5 but for the 10 children available in the UVA/Padova simulator, also for the $A R G$ and the $A R G_{A E}$ with $\beta=1.25$. In this case, hyperglycemia is increased when using the $A R G_{A E}$. However, hypoglycemia is avoided with the $A R G_{A E}$, which is the primary goal specially when regulating $\mathrm{BG}$ in children since it can have the most severe consequences. Additionally, as with adolescents, the lowest penalty index is achieved with the $A R G_{A E}$. 
Table 6 Results (\% of time) for the 10 children of the UVA/Padova Simulator using the ARG and $A R G_{A E}$ with $\beta=1.25$. Meal size: 70 gCHO (large). Announced meal size: Medium.

\begin{tabular}{lll}
\hline $\begin{array}{l}\text { Children } \\
\text { Underestimated Meal }\end{array}$ & $A R G$ & $A R G_{A E}$ \\
\hline Hyperglycemia & $24.0+-3.9$ & $26.0+-5.6$ \\
Hypoglycemia & $4.0+-6.5$ & $0.0+-0.0$ \\
Severe hyperglycemia & $17.6+-3.9$ & $17.8+-4.3$ \\
Desired range & $72.0+-5.5$ & $74.0+-5.6$ \\
Acceptable range & $78.3+-6.1$ & $82.2+-4.3$ \\
\hline Penalty Index & 14.74 & 12.30
\end{tabular}

3.2 in silico evaluation with unannounced meals

Table 7 Results (\% of time) for the 10 adolescents of the UVA/Padova Simulator using the $A R G, A R G_{80 \%}$ and $A R G_{A E}$ with different values of $\beta$. Meal size: 55 gCHO (medium). Meal size considered: Medium.

\begin{tabular}{llllll}
\hline $\begin{array}{l}\text { Adolescents } \\
\text { Unannounced 55g Meal }\end{array}$ & $A R G$ & $A R G_{80 \%}$ & $\begin{array}{l}A R G_{A E} \\
\beta=1.10\end{array}$ & $\begin{array}{l}A R G_{A E} \\
\beta=1.15\end{array}$ & $\begin{array}{l}A R G_{A E} \\
\beta=1.20\end{array}$ \\
\hline Hyperglycemia & $23.7+-4.2$ & $27.7+-4.8$ & $23.8+-4.1$ & $23.1+-4.0$ & $22.5+-4.0$ \\
Hypoglycemia & $1.7+-3.7$ & $0.0+-0.0$ & $0.0+-0.0$ & $0.0+-0.0$ & $0.3+-1.1$ \\
Severe hyperglycemia & $12.7+-5.3$ & $14.6+-5.7$ & $12.5+-5.3$ & $12.1+-5.2$ & $11.9+-5.2$ \\
$\begin{array}{l}\text { Desired range } \\
\text { Acceptable range }\end{array}$ & $74.6+-6.5$ & $72.3+-4.8$ & $76.2+-4.1$ & $76.9+-4.0$ & $77.2+-4.0$ \\
\hline Penalty Index & $85.6+-7.3$ & $85.4+-5.7$ & $87.5+-5.3$ & $87.9+-5.2$ & $87.8+-5.5$ \\
\hline
\end{tabular}

Table 8 Results (\% of time) for the 10 children of the UVA/Padova Simulator using the $A R G, A R G_{80 \%}$ and $A R G_{A E}$ with different values of $\beta$. Meal size: 55 gCHO (medium). Meal size considered: Medium.

\begin{tabular}{llllll}
\hline $\begin{array}{l}\text { Children } \\
\text { Unannounced 55g Meal }\end{array}$ & $A R G$ & $A R G_{80 \%}$ & $\begin{array}{l}A R G_{A E} \\
\beta=1.00\end{array}$ & $\begin{array}{l}A R G_{A E} \\
\beta=1.05\end{array}$ & $\begin{array}{l}A R G_{A E} \\
\beta=1.10\end{array}$ \\
\hline Hyperglycemia & $20.8+-3.1$ & $24.6+-4.2$ & $23.1+-4.6$ & $22.2+-4.3$ & $21.1+-3.9$ \\
Hypoglycemia & $6.3+-8.6$ & $1.3+-4.2$ & $0.0+-0.0$ & $0.4+-1.3$ & $4.5+-6.2$ \\
Severe hyperglycemia & $14.2+-3.5$ & $16.8+-3.9$ & $15.0+-3.9$ & $14.4+-3.7$ & $13.9+-3.7$ \\
$\begin{array}{l}\text { Desired range } \\
\text { Acceptable range }\end{array}$ & $72.9+-6.9$ & $74.0+-5.9$ & $76.9+-4.6$ & $77.4+-4.5$ & $74.4+-4.4$ \\
\hline Penalty Index & $79.5+-7.6$ & $81.9+-6.4$ & $85.0+-3.9$ & $85.1+-4.3$ & $81.6+-5.4$ \\
\hline
\end{tabular}

Tables 7 and 8 show the mean \pm 1 std of the $\%$ time in hyperglycemia $(>180 \mathrm{mg} / \mathrm{dl})$, hypoglycemia $(<70 \mathrm{mg} / \mathrm{dl})$, severe hyperglycemia $(>250 \mathrm{mg} / \mathrm{dl})$, the desired range $(70-180 \mathrm{mg} / \mathrm{dl})$ and the acceptable range $(70-250 \mathrm{mg} / \mathrm{dl})$ for the 10 adolescents available in commercial version of the UVA/Padova simulator using the $A R G$, the $A R G_{80 \%}$ and the $A R G_{A E}$ for a 55 gCHO meal when no meal announcement is used. As it was demonstrated for the adult population in [19], the ARG with unannounced meals achieves similar in silico results to the ARG with 
meal announcement. The $A R G_{A E}$ also preserves its performance, but using a different value of $\beta$. This is due to the fact that, in section 3.1, the IOB limit is increased at the time of meal announcement, whereas here it is raised when the meal is detected (i.e., when glucose values raise). Figure 8 shows this difference in the IOB constraints.

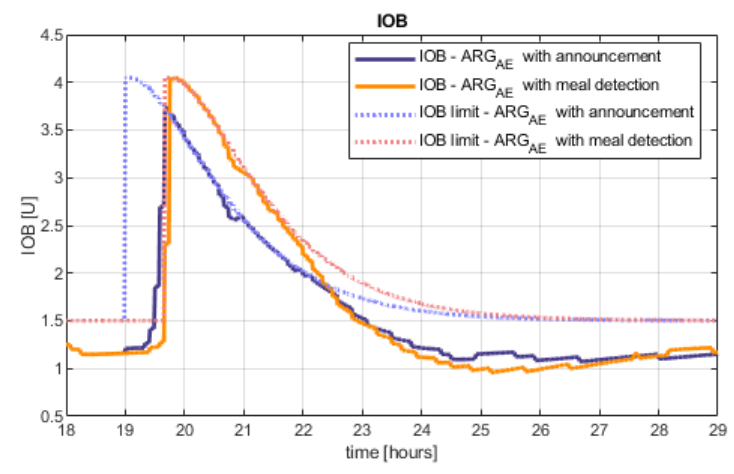

Fig. 8 IOB and IOB constraint with the $A R G_{A E}$ with meal announcement and the $A R G_{A E}$ with meal detection.

As was the case with announced meals, the $A R G_{A E}$ presents improved performance compared to both the ARG and $A R G_{80 \%}$. The same behaviour is obtained when considering over- (tables 9 and 10) and underestimated meals (tables 11 and 12). If meals are overestimated, the ARG and the $A R G_{A E}$ show similar performance. However, if a conservative $\beta$ is selected, hypoglycemia is reduced while maintaining low hyperglycemia. For example, for the adolescent population if $\beta=1.1$ is used: \% time in hyperglycemia $=18.5 \pm 3.9$, hypoglycemia $=1.9 \pm 6.1$, severe hyperglycemia $=4.4 \pm 5.9$, the desired range $=79.6 \pm 6.7$ and the acceptable range $=93.7 \pm 7.3$, yielding an index of 4.49 . For underestimated meals, the most adequate algorithm remains the $A R G_{A E}$.

Table 9 Results (\% of time) for the 10 adolescents of the UVA/Padova Simulator using the $A R G$ and $A R G_{A E}$ with $\beta=1.15$. Meal size: $40 \mathrm{gCHO}$ (small). Meal size considered: Medium.

\begin{tabular}{lll}
\hline $\begin{array}{l}\text { Adolescents } \\
\text { Unannounced 40g Meal }\end{array}$ & $A R G$ & $A R G_{A E}$ \\
\hline Hyperglycemia & $18.7+-3.9$ & $18.1+-3.9$ \\
Hypoglycemia & $5.7+-9.5$ & $5.1+-8.7$ \\
Severe hyperglycemia & $4.5+-6.0$ & $4.3+-5.8$ \\
Desired range & $75.6+-11.0$ & $76.8+-10.0$ \\
Acceptable range & $89.8+-11.1$ & $90.6+-9.8$ \\
\hline Penalty Index & 5.54 & 5.62
\end{tabular}


Table 10 Results (\% of time) for the 10 children of the UVA/Padova Simulator using the $A R G$ and $A R G_{A E}$ with $\beta=1.05$. Meal size: $40 \mathrm{gCHO}$ (small). Meal size considered: Medium.

\begin{tabular}{lll}
\hline $\begin{array}{l}\text { Children } \\
\text { Unannounced 40g Meal }\end{array}$ & $A R G$ & $A R G_{A E}$ \\
\hline Hyperglycemia & $16.6+-2.9$ & $16.9+-3.2$ \\
Hypoglycemia & $7.5+-10.1$ & $6.4+-8.7$ \\
Severe hyperglycemia & $8.5+-3.9$ & $8.5+-3.9$ \\
Desired range & $75.9+-8.1$ & $76.7+-6.5$ \\
Acceptable range & $84.1+-8.9$ & $85.1+-7.5$ \\
\hline Penalty Index & 10.75 & 9.00
\end{tabular}

Table 11 Results (\% of time) for the 10 adolescents of the UVA/Padova Simulator using the $A R G$ and $A R G_{A E}$ with $\beta=1.15$. Meal size: $70 \mathrm{gCHO}$ (large). Meal size considered: Medium.

\begin{tabular}{lll}
\hline $\begin{array}{l}\text { Adolescents } \\
\text { Unannounced 70g Meal }\end{array}$ & $A R G$ & $A R G_{A E}$ \\
\hline Hyperglycemia & $28.1+-4.1$ & $27.9+-4.2$ \\
Hypoglycemia & $1.2+-3.9$ & $0.0+-0.0$ \\
Severe hyperglycemia & $18.4+-4.4$ & $18.0+-4.5$ \\
Desired range & $70.7+-7.0$ & $72.1+-4.2$ \\
Acceptable range & $80.4+-7.3$ & $82.0+-4.5$ \\
\hline Penalty Index & 11.46 & 10.93
\end{tabular}

Table 12 Results (\% of time) for the 10 children of the UVA/Padova Simulator using the $A R G$ and $A R G_{A E}$ with $\beta=1.05$. Meal size: $70 \mathrm{gCHO}$ (large). Meal size considered: Medium.

\begin{tabular}{lll}
\hline $\begin{array}{l}\text { Children } \\
\text { Unannounced 70g Meal }\end{array}$ & $A R G$ & $A R G_{A E}$ \\
\hline Hyperglycemia & $24.7+-3.5$ & $27.5+-5.4$ \\
Hypoglycemia & $4.6+-7.2$ & $0.0+-0.0$ \\
Severe hyperglycemia & $18.8+-3.6$ & $19.6+-4.2$ \\
Desired range & $70.7+-6.3$ & $72.5+-5.4$ \\
Acceptable range & $76.6+-6.9$ & $80.4+-4.2$ \\
\hline Penalty Index & 16.58 & 14.11
\end{tabular}

\section{Discussion}

Intensive in silico studies show that a simple retune of the $\overline{I O B}$ is not enough to achieve satisfactory performance in children and adolescents using the ARG algorithm. When the meal size is estimated properly, the $A R G_{A E}$ has the ability of reducing hypoglycemia episodes as was done with the $A R G$ with reduced IOB constraint but without increasing (or even further lowering) time in hyperglycemia compared to the $A R G$. Thus, the compromise between prandial hyperglycemia and postprandial hypoglycemia is reduced.

When meals are not classified adequately, the $A R G_{A E}$ shows consistent robust performance in children and adolescents. When meals are overestimated, hypoglycemia might not be reduced compared to the ARG if $\beta$ is too relaxed. However, $\beta$ can be as conservative as necessary taking into account individual patient habits, achieving effective hypoglycemia diminution.

When no meal announcement is used, the $A R G_{A E}$ performance is consistent with the announced-meal scenario when an adequate value of $\beta$ is selected, achiev- 
ing the better outcome from both algorithms. However, as expected, for both the $A R G_{A E}$ and the $A R G$, time in the desired and the acceptable ranges are reduced compared with their versions using meal announcement. This is due to the additional delay in the aggressive controller action introduced by the meal detection algorithm. Since no meal announcement is used, the algorithm must check additional conditions (the ones predefined for $g_{f}$ and $Q_{s t o 1}$ ) in order to verify the appearance of a meal.

Another important upside of the $A R G_{A E}$ is that its tuning is relative to the open-loop therapy. This is an advantage since it is more user-friendly, and can use the information from the traditional patient treatment.

Lastly, it must be kept in mind that no extra protection layers were used in this in silico study. More tests must be carried out using hypo- and hyperglycemia protection layers as the ones employed in [12] in order to further improve the controller performance.

\section{Conclusions}

A strategy for automatic glycemic regulation based on switched control and timevarying IOB constraints was introduced and compared with the previously tested ARG algorithm. The comparison is made in silico considering the pediatric population available in the UVA/Padova simulator, on account of the coming up clinical trials in children and adolescents. Simulations show that the proposed algorithm improves the performance of the ARG algorithm and is robust to misclassified meals, as well as providing intuitive tuning. The scenario considering unannounced meals is also explored, accomplishing consistent performance from the $A R G_{A E}$. Thus, future in vivo testing of the ARG algorithm will involve the $A R G_{A E}$ configuration.

Acknowledgements Research in this area is supported by the Argentine government (PICT 2017-3211 Agencia Nacional de Promoción Científica y Tecnológica, PIP 112-201501-00837 CONICET, and UNLP 11/I216)

\section{References}

1. A. Haidar, "The artificial pancreas: How closed-loop control is revolutionizing diabetes," IEEE Control Systems, vol. 36, no. 5, pp. 28-47, Oct 2016.

2. B. Bequette, "Challenges and recent progress in the development of a closed-loop artificial pancreas," Annu Rev Control, vol. 36, pp. 255-266, 2012.

3. D. Shi, E. Dassau, and F. J. Doyle, "Adaptive zone model predictive control of artificial pancreas based on glucose- and velocity-dependent control penalties," IEEE Transactions on Biomedical Engineering, vol. 66, no. 4, pp. 1045-1054, 2019.

4. A. Abitbol et al., "Overnight glucose control with dual- and single-hormone artificial pancreas in type 1 diabetes with hypoglycemia unawareness: A randomized controlled trial," Diabetes Technology ES Therapeutics, vol. 20, no. 3, pp. 189-196, Mar. 2018.

5. L. Bally et al., "Day-and-night glycaemic control with closed-loop insulin delivery versus conventional insulin pump therapy in free-living adults with well controlled type 1 diabetes: An open-label, randomised, crossover study," Lancet Diabetes Endocrinol, vol. 5, no. 4, pp. 261-270, Apr 2017.

6. G. Steil, "Algorithms for a closed-loop artificial pancreas: The case for proportionalintegral-derivative control," J Diabetes Sci Technol, vol. 7, no. 6, pp. 1621-1631, Nov 2013. 
7. T. Ly et al., "Day and night closed-loop control using the integrated Medtronic hybrid closed-loop system in type 1 diabetes at diabetes camp," Diabetes Care, vol. 38, no. 7, pp. 1205-1211, Jul 2015.

8. A. Beneyto and J. Vehi, "Postprandial fuzzy adaptive strategy for a hybrid proportional derivative controller for the artificial pancreas," Medical \&5 Biological Engineering 85 Computing, vol. 56, no. 11, pp. 1973-1986, Nov. 2018.

9. R. Mauseth et al., "Use of a "fuzzy logic" controller in a closed-loop artificial pancreas," Diabetes Technology and Therapeutics, vol. 15, no. 8, pp. 628-633, 82013.

10. R. S. Sánchez-Peña and D. R. Cherñavvsky, The Artificial Pancreas: Current Situation and Future Directions. Academic Press, 2019.

11. A. S. Brazeau et al., "Carbohydrate counting accuracy and blood glucose variability in adults with type 1 diabetes," Diabetes Res Clin Pract, vol. 99, no. 1, pp. 19-23, Jan 2013.

12. R. Sánchez-Peña et al., "Artificial pancreas: Clinical study in Latin America without premeal insulin boluses," J Diabetes Sci Technol, vol. 12, no. 5, pp. 914-925, 2018.

13. P. Colmegna et al., "Automatic regulatory control in type 1 diabetes without carbohydrate counting," Control Eng Pract, pp. 22-32, 2018.

14. A. Revert et al., "Safety auxiliary feedback element for the artificial pancreas in type 1 diabetes," IEEE Trans Biomed Eng, vol. 60 (8), pp. 2113-2122, 2013.

15. S. W. Powers et al., "Parent report of mealtime behavior and parenting stress in young children with type 1 diabetes and in healthy control subjects," Diabetes Care, vol. 25, no. 2, p. 313, Feb. 2002.

16. K. Turksoy et al., "Real-time insulin bolusing for unannounced meals with artificial pancreas," Control Eng Pract, vol. 59, pp. 159-164, 2017.

17. F. M. Cameron et al., "Closed-loop control without meal announcement in type 1 diabetes," Diabetes technology \&6 therapeutics, vol. 19, no. 9, pp. 527-532, Sep. 2017.

18. H. Blauw et al., "Performance and safety of an integrated bihormonal artificial pancreas for fully automated glucose control at home," Diabetes, obesity $\&$ metabolism, vol. 18, no. 7 , pp. 671-677, Jul. 2016.

19. E. Fushimi et al., "Artificial pancreas: Evaluating the arg algorithm without meal announcement," J Diabetes Sci Technol, p. 1932296819864585, Jul. 2019

20. —_, "Artificial pancreas clinical trials: Moving towards closed-loop control using insulinon-board constraints," Biomed Signal Process Control, vol. 45, pp. 1-9, Aug. 2018.

21. L. DeJournett, "Essential elements of the native glucoregulatory system, which, if appreciated, may help improve the function of glucose controllers in the intensive care unit setting," J Diabetes Sci Technol, vol. 4, no. 1, pp. 190-198, Jan. 2010. [Online]. Available: https://doi.org/10.1177/193229681000400124

22. C. Dalla Man, R. Rizza, and C. Cobelli, "Meal simulation model of the glucose-insulin system," IEEE Trans Biomed Eng, vol. 54, no. 10, pp. 1740-1749, 2007.

23. G. C. Goodwin et al., "A fundamental control limitation for linear positive systems with application to type 1 diabetes treatment," Automatica, vol. 55, pp. 73-77, May 2015.

24. B. Kovatchev, "Glycemic variability: Risk factors, assessment, and control," $J$ Diabetes Sci Technol, vol. 13, no. 4, pp. 627-635, Jul. 2019. [Online]. Available: https://doi.org/10.1177/1932296819826111 


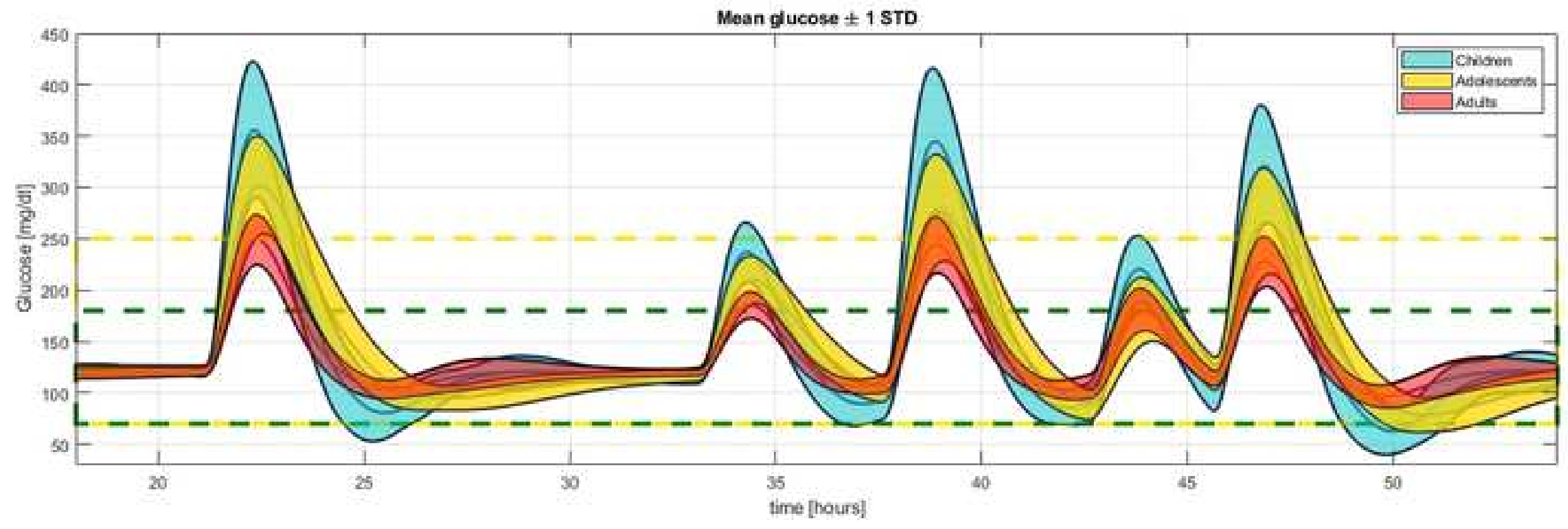



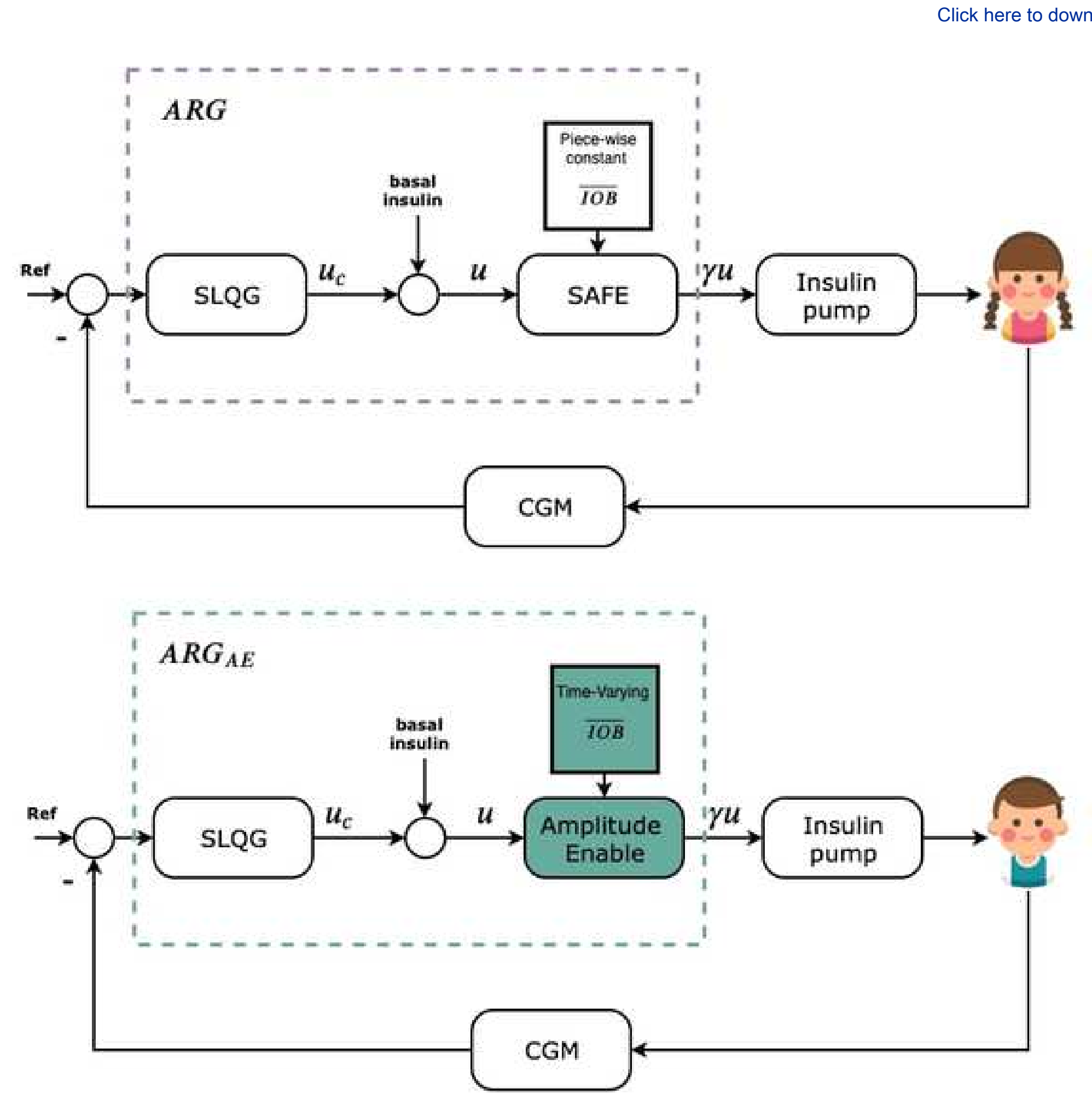


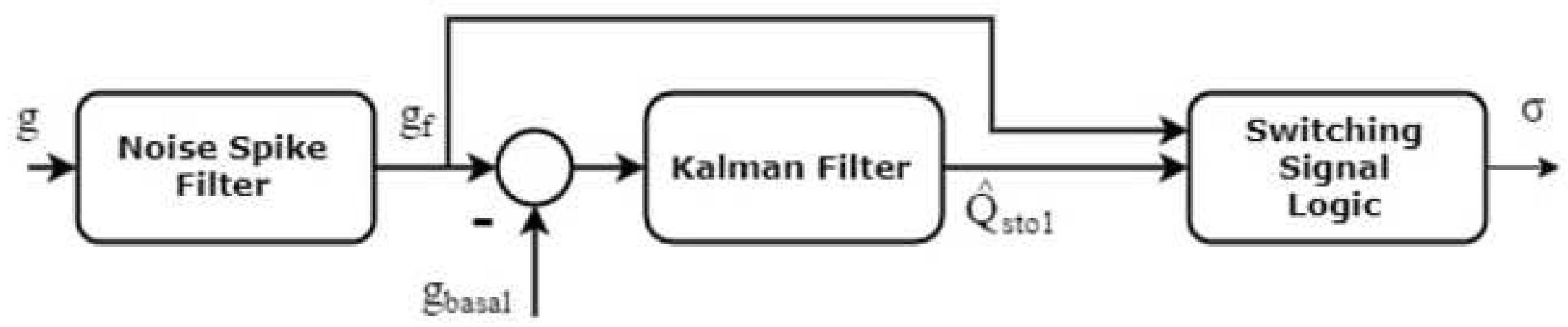




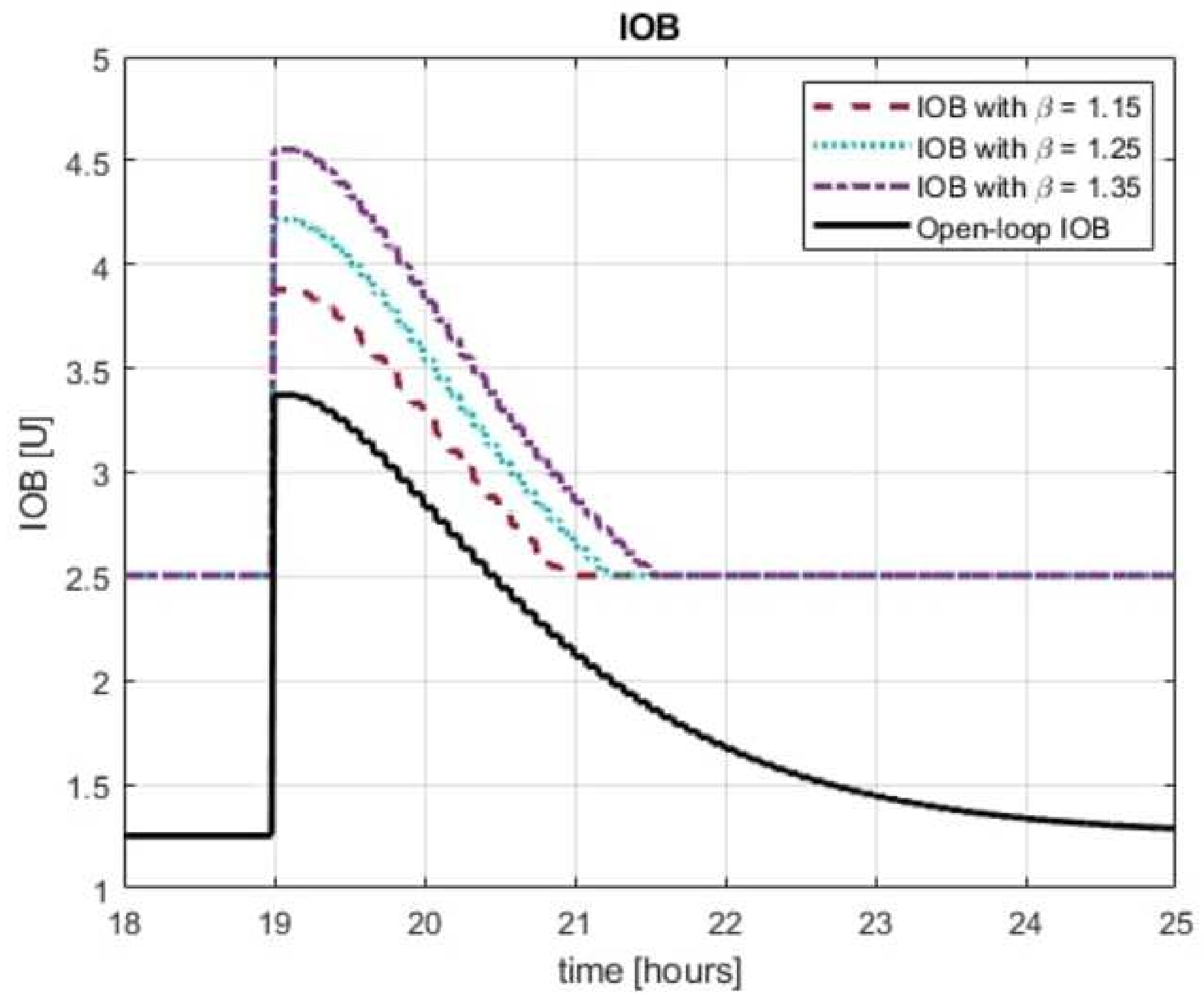




\section{Subcutaneous insulin}
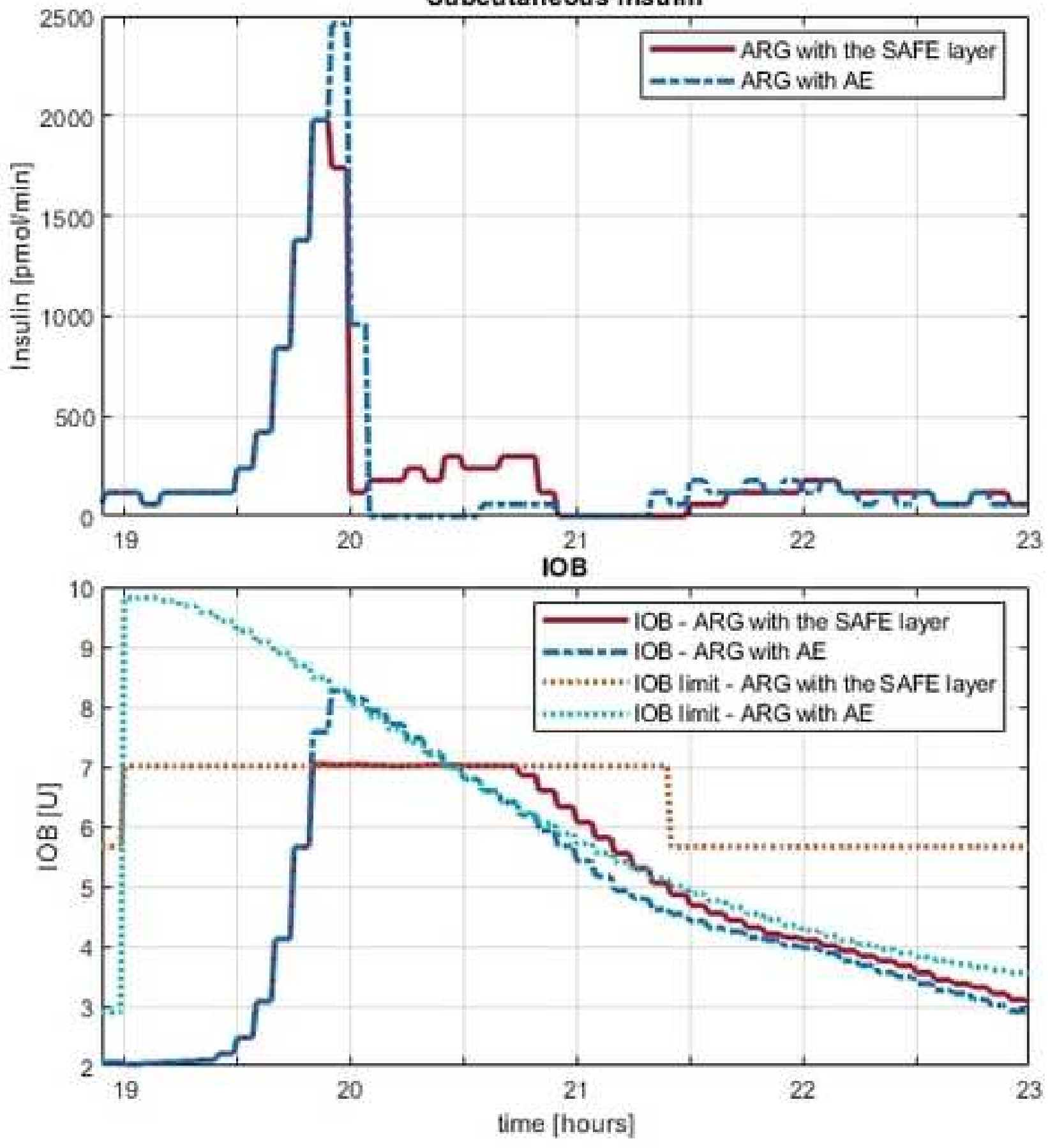


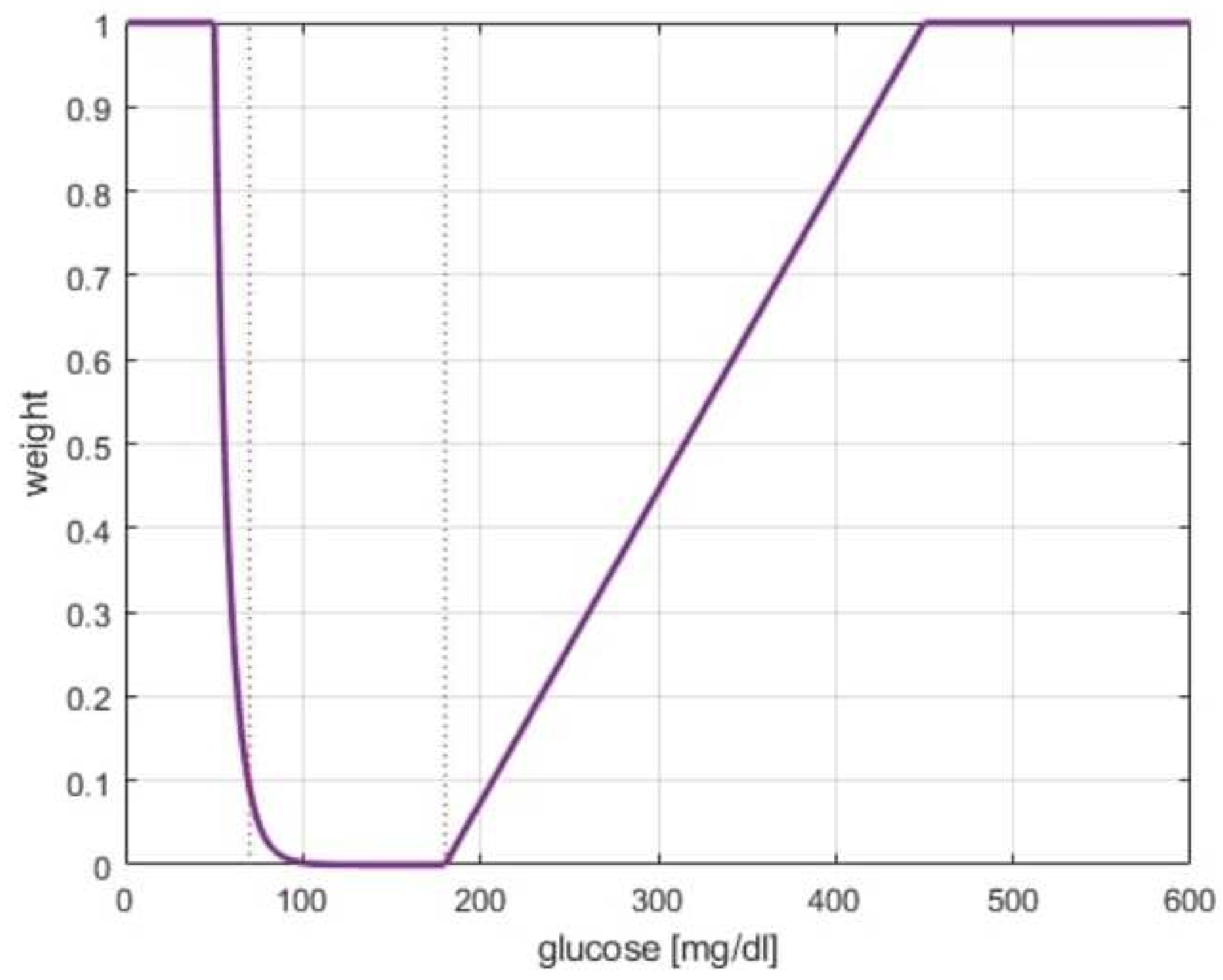



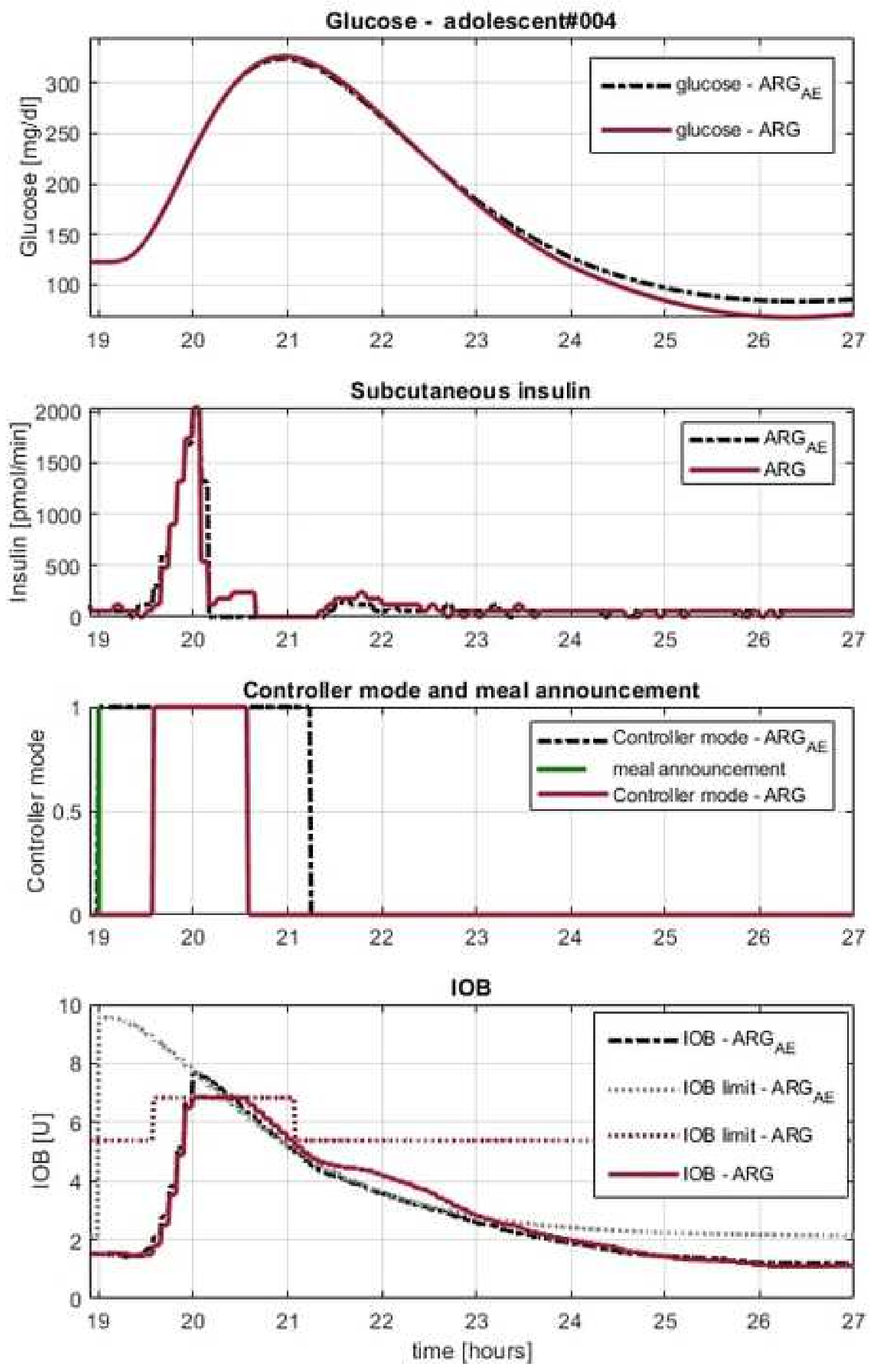


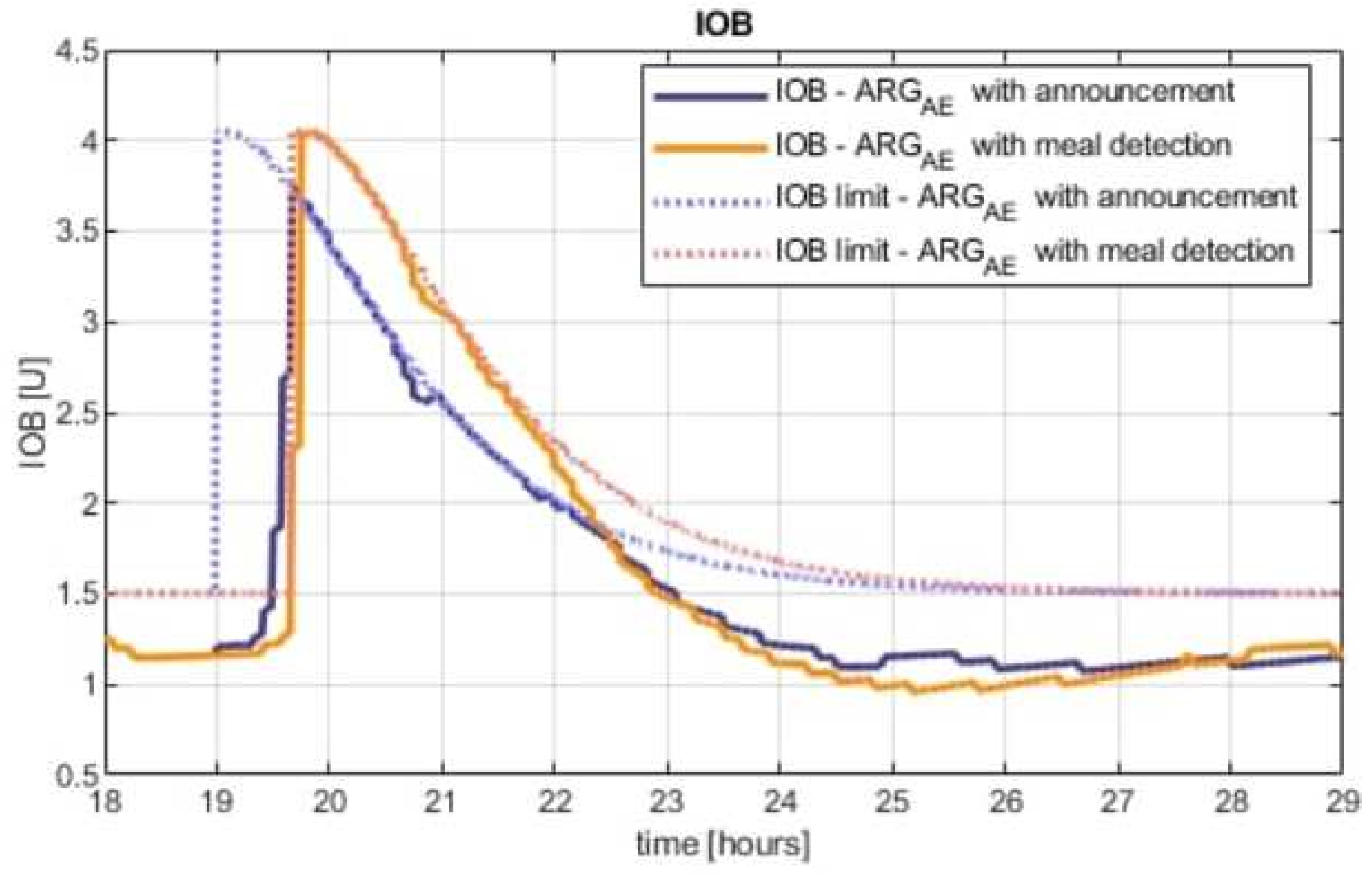



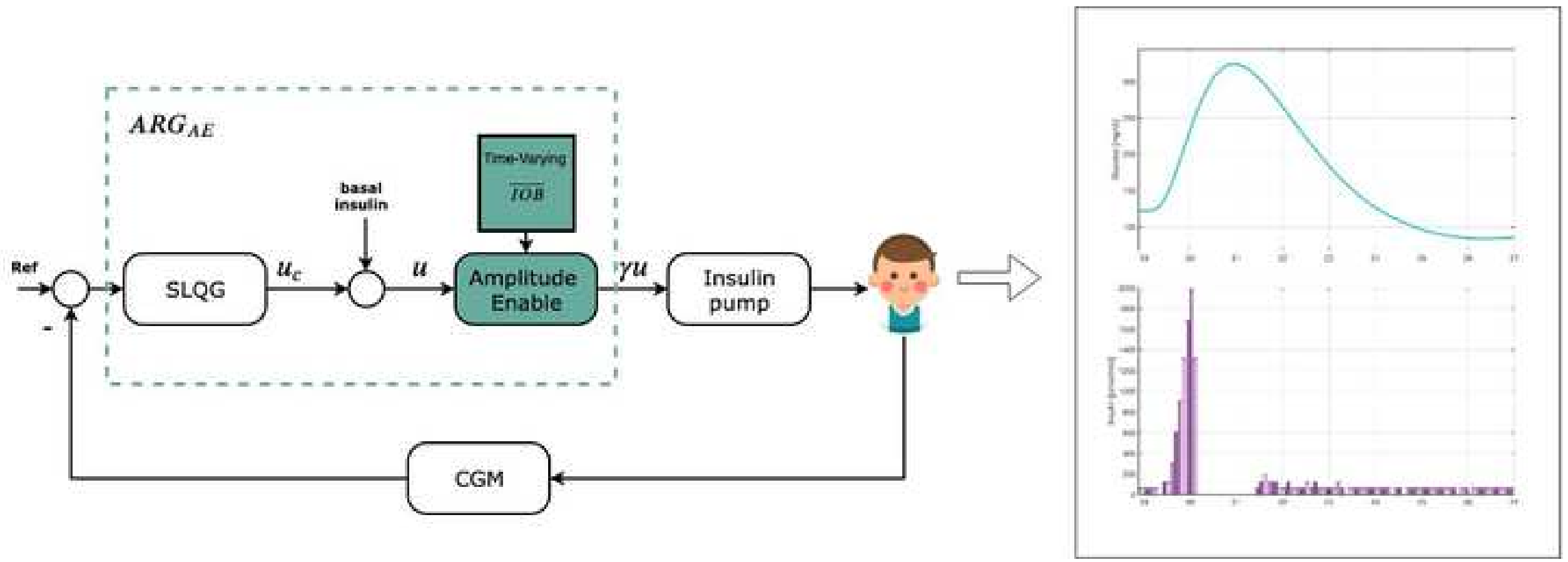